FORMATION Formation emploi

Revue française de sciences sociales

104 | octobre-décembre 2008

Pêle-mêle

\title{
Formation professionnelle continue en Belgique : des pratiques variables selon les trajectoires professionnelles
}

Continuous vocational training in Belgium: variable practices depending on the career pathway

Berufliche Weiterbildung in Belgien: unterschiedliche Praktiken je nach beruflichem Werdegang

Formación profesional continua en Bélgica : prácticas variables según las

trayectorias profesionales

\section{Bernard Conter et Matthieu Veinstein}

\section{OpenEdition}

Journals

Édition électronique

URL : http://journals.openedition.org/formationemploi/1362

DOI : 10.4000/formationemploi.1362

ISSN : 2107-0946

Éditeur

La Documentation française

Édition imprimée

Date de publication : 1 octobre 2008

Pagination : 67-82

ISSN : 0759-6340

Référence électronique

Bernard Conter et Matthieu Veinstein, «Formation professionnelle continue en Belgique : des pratiques variables selon les trajectoires professionnelles », Formation emploi [En ligne], 104 | octobredécembre 2008, mis en ligne le 01 octobre 2010, consulté le 30 octobre 2020. URL : http:// journals.openedition.org/formationemploi/1362 ; DOI : https://doi.org/10.4000/formationemploi.1362 


\section{Pays étrangers}

\section{Formation professionnelle continue en Belgique : des pratiques variables selon les trajectoires professionnelles ${ }^{1}$}

Par Bernard Conter et Matthieu Veinstein*

Les pratiques de formation professionnelle continue des travailleurs sont très différenciées. Les travailleurs les mieux insérés accèdent plus souvent aux formations, même s'il s'agit souvent de formations courtes. À l'inverse,

les formations organisées dans le cadre de programmes d'emploi sont moins fréquentes mais plus longues.

Le développement de la formation continue constitue une des priorités de l'agenda politique européen et national en Belgique. Les enquêtes ou indicateurs réalisés pour mesurer l'accroissement des pratiques de formation en offrent en général une vue statique et portent sur une année civile (voire moins).

L'enquête sur le Panel belge des ménages (encadré 1), réalisée annuellement, offre la possibilité d'aborder la question de la formation selon une approche longitudinale. Nous avons exploité les quelques données relatives à l'emploi et à la formation de cette enquête générale pour aborder deux questions.

\footnotetext{
${ }^{1}$ Le présent article constitue la prolongation d'un travail réalisé en vue des Journées du Longitudinal 2005 (Cardelli et al., 2005). Nous remercions Rébecca Cardelli pour sa précieuse collaboration.
}

La première porte sur la participation et la participation répétée à la formation professionnelle (que nous

* Bernard Conter est attaché scientifique à l'Institut wallon de l'évaluation, de la prospective et de la statistique (IWEPS). Ses recherches sont essentiellement consacrées aux effets et aux transformations des politiques de I'emploi et de la formation. II a récemment coordonné, avec Ph. Lemistre et B. Reynes, l'ouvrage L'ancienneté professionnelle à l'épreuve de la flexicurité là paraître aux Presses Universitaires des Sciences Sociales de Toulouse).

Matthieu Veinstein est chercheur au centre METICES TEF Migrations Espaces Travail Institutions Citoyenneté Épistémologie Santé - Travail Emploi Formation) de l'université Libre de Bruxelles (ULB). Ses travaux récents portent sur l'insertion professionnelle des jeunes. 
avons qualifiée de « récurrence ») sur une période de cinq ans (1997-2001). À partir des données disponibles sur le type de formation suivie (initiative, durée, financement), nous avons distingué différentes « pratiques de formation » et observé, en cas de récurrence, si ces pratiques étaient diverses ou homogènes.

Dans un deuxième temps, nous avons tenté de mettre en perspective les pratiques de formation avec les trajectoires professionnelles des individus composant notre échantillon. Nous postulions en effet que la position sur le marché du travail mais aussi la stabilité de l'insertion étaient déterminantes de l'accès et des pratiques de formation.
Enfin, nous avons cherché à distinguer ces pratiques au sein du groupe d'individus, majoritaires dans l'échantillon, dont les trajectoires renvoient à une stabilisation en emploi sur la période étudiée. En effet, en dépit de ce trait commun, ces trajectoires regroupent des situations d'emploi très contrastées. Elles concernent tant des travailleurs (travailleuses) à temps partiel et bas salaires que des cadres supérieurs d'entreprise. On peut s'attendre à ce que ces conditions d'emplois soient associées à des rythmes et pratiques de formation différenciés.

\section{Encadré 1 \\ Le Panel Survey on Belgian Household (PSBH) et l'échantillon choisi}

Le Panel belge des ménages est une enquête récurrente réalisée en Belgique depuis 1992 ; elle a été intégrée à l'ECHP (*) en 1994. Le panel se compose de 3500 ménages et s'adresse à 6500 adultes

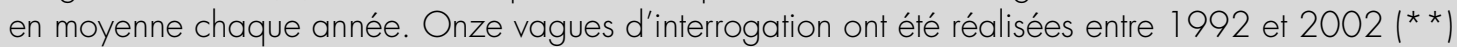

Nous avons choisi de travailler sur les individus de 18 à 65 ans ayant participé sans interruption aux cinq dernières vagues de ce panel, à savoir celles réalisées au cours des années 1998 à 2002 et portant sur l'année civile précédente. Nous avons ainsi pris en compte 3509 individus dont les données sont complètes pour les années 1997 à $2001\left(^{* \star *}\right)$.

Cet échantillon se compose de $47 \%$ d'hommes et de 53 \% de femmes. Les diplômés de l'enseignement primaire ou secondaire inférieur représentent $27 \%$ de l'échantillon pour $34 \%$ de diplômés de l'enseignement secondaire supérieur et $39 \%$ de diplômés de l'enseignement supérieur. Si l'on observe la répartition régionale du domicile, on compte $8 \%$ d'individus habitant la Région de Bruxelles-Capitale, 58 \% la Flandre et 32 \% la Wallonie. Au moment de l'enquête en 2002, on compte 71 \% d'individus en emploi pour $7 \%$ de chômeurs et 22 \% d'inactifs.

La comparaison de la structure de cet échantillon avec les données issues de l'Enquête sur les forces de travail (EFT, 2002) indique une composition relativement semblable, en termes de sexe, d'âge, de région et de statut professionnel, à l'ensemble de la population belge. Cependant, certaines catégories sont surreprésentées dans le panel : il s'agit, pour l'essentiel, des individus de la tranche d'âge 3544 ans, des personnes ayant un emploi et des diplômés de l'enseignement supérieur.

(*) : L'European Community Household Panel est l'équivalent du PSBH au niveau européen. L'objectif de l'étude ECHP est toutefois plus restreint car il se concentre sur l'activité économique et le revenu des ménages.

$(* *)$ : Sur les travaux réalisés à partir du PSBH, voir Doutrelepont et al., 2004.

$(* * *)$ : Le choix d'une période de cinq années a été opéré en raison des phénomènes d'attrition. Si nous avons sélectionné notre échantillon au départ de la vague 7 (1998) plutôt que de la vague 1, c'est en raison d'une part, des modifications survenues dans l'enquête en 1994 (les données PSBH ont été incorporées dès la troisième vague à l'ECHP afin d'établir des comparaisons à un niveau européen) et, d'autre part, de la recharge de l'échantillon en 1998 (860 nouveaux ménages ont été ainsi ajoutés à l'échantillon initial). 


\section{LES PRATIQUES DE FORMATION PROFESSIONNELLE CONTINUE EN BELGIQUE}

\section{Éléments de contexte}

La formation continue est développée par plusieurs types d'acteurs s'appuyant sur différentes sources de financement. La relative extension des pratiques, publics et sources de financement, combinée à une volonté croissante de régulation publique ont contribué à institutionnaliser un champ de la formation caractérisé par une certaine mixité des fonctions (Conter, Maroy, 1999). La distinction des opérateurs selon les types de publics pris en charge (travailleurs, demandeurs d'emploi, jeunes en alternance, publics fragilisés, etc.) devient de moins en moins opérationnelle.

Parmi les principaux opérateurs, l'enseignement de promotion sociale offre des formations diplômantes, tandis que les services publics de l'emploi assurent la formation des demandeurs d'emploi et des travailleurs dans une perspective davantage liée à l'exercice du métier, tout comme les fonds de formation sectoriels gérés par les interlocuteurs sociaux ${ }^{2}$. Le secteur privé développe une offre de formation pour les travailleurs mais il est difficile d'en réaliser un inventaire. Les entreprises participent au financement de la formation via une cotisation obligatoire (d'au moins $0,1 \%$ de la masse salariale) à un fonds sectoriel. En outre, les interlocuteurs sociaux se sont engagés à consacrer $1,9 \%$ de la masse salariale à la formation (Monville, 2007), sans toutefois atteindre l'objectif à ce jour ${ }^{3}$. Pour encourager la formation des travailleurs, les pouvoirs publics soutiennent le dispositif du congé éducation payé qui prévoit, outre le droit de s'absenter, un remboursement plafonné du coût salarial durant les heures de formation. De même, les régions ont développé un système de

\footnotetext{
${ }^{2}$ On utilise en Belgique traditionnellement l'expression d' $"$ interlocuteurs sociaux » pour désigner les acteurs de la concertation sociale. Le terme de "partenaires » a été introduit en Belgique plus tardivement, notamment à la suite du lancement du « dialogue social européen » par J. Delors.

${ }^{3}$ Les dépenses de formation des entreprises s'élèvent à $1,08 \%$ de la masse salariale en 2006; elles atteignaient $1,34 \%$ en 2000 (Monville et Léonard, 2008).
}

chèque formation à destination des employeurs. Pour les demandeurs d'emploi peu qualifiés, différents dispositifs d'insertion professionnelle complètent l'offre. De plus en plus, ils articulent une période de stage. Ajoutons à ce bref inventaire, les centres de formation en alternance dans l'enseignement et un institut organisant la formation des apprentis aux métiers d'indépendants ${ }^{4}$.

La multiplicité des dispositifs de formation en Belgique et la rareté des instruments statistiques rendent complexe l'étude de tels dispositifs. Les sources les plus fréquemment utilisées pour mesurer l'accès à la formation sont l'Enquête sur les forces de travail (EFT) (Baye et al., 2003) et l'enquête Continual Vocational Training Survey (CVTS) s'adressant aux employeurs (Nestler \& Kailis, 2002 ; De Brier \& Legrain, 2002). Le Bilan social permet par ailleurs d'évaluer la proportion de salariés en formation dans les entreprises (Heuse \& Zachary, 2003) .

Ces travaux situent la Belgique sous la moyenne des pays européens en ce qui concerne la participation des individus ou des salariés à la formation. Ils soulignent aussi, s'agissant du pays et, parfois, de ses régions, une variation importante de l'accès à la formation continue liée à plusieurs caractéristiques d'ordre personnel ou structurel. Ainsi, le sexe, le niveau de diplôme, la situation professionnelle, l'âge, la région ou le secteur professionnel apparaissent comme des facteurs discriminants.

Ces différentes enquêtes ont également permis d'établir deux constats généraux: celui d'une augmentation progressive des niveaux de participation à la formation en Belgique (même si le retournement conjoncturel du début des années 2000 semble avoir rompu la tendance), et celui d'une progressive diminution des écarts entre groupes sociaux ou professionnels. De Brier et Legrain (2002), qui ont comparé les résultats de CVTS avec des enquêtes similaires réalisées en 1986 et 1993, évoquent ainsi une "démocratisation de l'accès à la formation » des salariés, au sens où les employés et les ouvriers

\footnotetext{
${ }^{4}$ On trouvera quelques repères historiques et statistiques sur la formation continue en Belgique dans Monville et Léonard, 2008.

${ }^{5}$ Pour une synthèse des différentes sources permettant d'évaluer le niveau de participation des adultes à la formation continue en Belgique francophone, voir Conter et al., 1999.
} 
participent plus que par le passé à la formation et où l'écart de participation entre ceux-ci et les cadres, bien que restant très important, tend à diminuer ${ }^{6}$.

L'objectif de notre travail était de poursuivre ces analyses par l'exploitation d'une source rarement

\footnotetext{
${ }^{6}$ Ce rappel des principaux résultats mis en évidence en Belgique ne doit pas occulter l'importance des éléments de contexte qui les déterminent largement. On peut citer à ce titre l'impact des stratégies des acteurs sociaux, travailleurs, employeurs, pouvoirs publics, souligné par Dubar (1990, p. 77), dans la structuration de l'offre de formation continue. Dans le cadre de contraintes économiques et sociales spécifiques, ces stratégies contribuent notamment à une structuration de l'offre de formation selon le secteur d'activité et la taille des entreprises, qui conditionne largement la participation aux formations continues sur les différents segments productifs.
}

utilisée pour l'étude de la participation à la formation professionnelle, le Panel des ménages belges (encadré 1). Cette enquête annuelle, qui n'est plus reconduite aujourd'hui, porte essentiellement sur les conditions de vie des individus (revenu, santé, logement, loisirs) ; elle aborde également les questions de l'emploi et de la participation à la formation professionnelle. Si l'enquête comporte peu de questions sur la formation (encadré 2), elle peut toutefois être utilisée dans une perspective longitudinale. Nous avons ainsi analysé cinq vagues du panel (1997 à 2001).

Sur les 3509 individus suivis dans le cadre de notre travail, 1330 ont participé à au moins une formation

\section{Encadré 2 \\ La formation continue dans le Panel Survey on Belgian Household}

Quelques questions du Panel belge des ménages abordent la participation à la formation continue. La question principale porte sur la participation au cours de l'année civile écoulée. Les formations visées s'apparentent, selon l'énoncé du questionnaire, à des cours, dans la mesure où "les formations informelles sur le lieu de travail, les instructions données par des collègues ou des supérieurs, l'apprentissage par soi-même, les compétences acquises grâce à l'exercice d'une profession » ne sont pas pris en compte, comme le précise le questionnaire d'enquête. Ces formations sont par ailleurs professionnelles au sens où " les cours d'intérêt général, c'est-à-dire ceux qui ne sont pas destinés à améliorer les compétences en vue de l'exercice d'une activité professionnelle particulière, ne sont pas inclus ". Quatre questions nous renseignent sur les modalités organisationnelles de la formation suivie. La première porte sur la durée, la deuxième sur le cadre dans lequel la formation a été suivie (en établissement scolaire ou universitaire, sur le lieu de travail, en alternance, dans le cadre d'un programme d'emploil ; enfin, il est demandé si les cours ont été payés ou organisés par l'employeur et s'ils ont été organisés à temps plein ou à temps partiel.

Les répondants ont aussi été questionnés sur les motivations et effets de la formation : celle-ci a-t-elle été suivie pour accroître des compétences ou des opportunités professionnelles et dans quelle mesure ces cours ont-ils été utiles en pratique pour atteindre ces deux objectifs? L'objet et la formulation de ces deux questions conduisent très souvent à une réponse affirmative, ce qui en limite l'intérêt.

Les questions posées à chaque vague du panel renvoient à une formation de référence. La prise en compte de plusieurs vagues de panel permet de voir, aux différentes vagues, si au moins une formation a été suivie.

En revanche, le panel ne permet pas de connaître le nombre de formations suivies au cours d'une année civile (associée à une vague du panel) $\left.\right|^{*}$ ).

$\left({ }^{*}\right)$ : Les possibilités de suivi longitudinal de la participation à la formation continue sont donc sensiblement différentes de celles offertes par l'enquête française «formation continue ». À partir de cette dernière, Trautman (2003) proposait une réflexion sur la « formation tout au long de la vie ». Il s'appuyait sur une connaissance du nombre de formations suivies depuis l'entrée dans la vie active et sur une description des formations suivies sur 14 mois (1999 et début 2000). 
au cours des cinq années considérées, soit $39 \%$. Cet accès à la formation diffère selon les facteurs évoqués plus haut (sexe, niveau de diplôme, situation professionnelle, âge) ${ }^{7}$.

\section{Diversité des formations suivies}

Une première étape de notre travail a été de déterminer le type de formation suivie par les participants à l'enquête. Bien que les formes que peut revêtir la formation continue soient multiples, le champ couvert par le Panel des ménages est celui des formations « en lien avec l'exercice d'une activité professionnelle particulière » (encadré 2).

L'enquête sur le Panel comporte peu de questions sur les modalités concrètes de la formation (encadré 2). Nous avons cependant cherché, à partir de l'association des variables existantes, à distinguer différentes pratiques de formation.

Il est d'abord possible de distinguer les formations sur une base statique (formations suivies en 2001), en tenant compte de certaines caractéristiques comme la durée, le cadre dans lequel la formation a été suivie, l'implication de l'employeur en termes d'organisation et/ou de financement. Les caractéristiques des formations varient peu d'une année sur l'autre.

La moitié des formations suivies ont une durée inférieure à la semaine et $70 \%$ n'excèdent pas quinze jours. Les formations les plus nombreuses avaient

${ }^{7}$ Les données sont présentées dans Cardelli et al (2005). lieu soit uniquement dans l'entreprise et étaient organisées ou payées par l'employeur $(43 \%)^{8}$, soit dans un établissement d'enseignement (40\%) et étaient alors dans la moitié des cas financées par l'employeur. Les formations «dans le cadre d'un système alterné $\rangle^{9}$ et les formations à destination des demandeurs d'emploi représentent, ensemble, $18 \%$ des formations.

Pour la suite de notre travail nous avons distingué parmi les deux premières catégories de formation du tableau 1 (formations exclusivement sur le lieu de travail et formations dans un établissement scolaire ou universitaire) d'une part les formations «avec implication de l'employeur » qui sont organisées et/ ou financées par l'employeur, et/ou se déroulent sur le lieu de travail, et d'autre part les formations que nous avons qualifiées de "formations d'initiative

${ }^{8}$ L'enquête ne permet pas de préciser si les formations non financées par l'employeur sont supportées par les pouvoirs publics ou par les individus.

${ }^{9}$ Le panel envisage les «formations professionnelles dans le cadre d'un système alterné comprenant un enseignement sur le lieu de travail et dans un établissement scolaire, y compris l'apprentissage». Cette catégorie regroupe donc des pratiques diverses, qui ne renvoient pas forcément à une pédagogie de l'alternance. En Belgique, la formation en alternance est essentiellement organisée dans le cadre de l'enseignement professionnel et de la scolarité obligatoire. La scolarité est obligatoire jusqu'à 18 ans, mais elle peut être accomplie en alternance à partir de 15 ans. Ce type de scolarité reste marginal au sein du système éducatif belge et, de manière plus générale, l'alternance est une pratique peu développée en Belgique (pour la seule Communauté française de Belgique, on compte 8700 jeunes en formation en alternance, soit $2,5 \%$ des effectifs de l'enseignement secondaire en 2006-2007).

Tableau 1

Caractéristiques des formations suivies en 2001

\begin{tabular}{|l|c|c|}
\hline \multicolumn{1}{|c|}{ Cadre institutionnel de formation suivie } & $\%$ & $\begin{array}{c}\text { Part des formations } \\
\text { payées ou organisées par } \\
\text { I'employeur (\%) }\end{array}$ \\
\hline Formation professionnelle exclusivement sur le lieu de travail & 43 & 96 \\
\hline Formation dans un établissement scolaire ou universitaire & 40 & 55 \\
\hline Autres formations en rapport avec des programmes d'emploi & 13 & $43^{\star}$ \\
\hline Formation alternée (lieu de travail et établissement scolaire) & 5 & 85 \\
\hline Total & $\mathbf{1 0 0}$ & $\mathbf{7 3}$ \\
\hline
\end{tabular}

Lecture : Parmi les formations suivies en 2001, $43 \%$ étaient réalisées sur un lieu de travail ; $96 \%$ des formations de ce type étaient organisées ou payées par l'employeur.

Note : Un nombre croissant de dispositifs publics ou visant l'insertion comprend des stages en entreprise.

Source : PSBH 2002. 
personnelle » dans la mesure où elles ne sont ni organisées, ni financées par un employeur, et ne se déroulent pas sur le lieu de travail.

\section{Approche longitudinale}

Pour appréhender la participation à la formation sur une base longitudinale, nous nous sommes d'abord attachés à prendre en compte la récurrence des participations à la formation sur la période.

Des différences apparaissent si l'on étudie cette récurrence, c'est-à-dire le nombre d'années au cours desquelles un individu a bénéficié de la formation. Ainsi, si $39 \%$ des individus suivent au moins une formation sur la période, $16 \%$ participent à la formation au cours d'une seule des cinq années, pour $11 \%$ au cours de deux années, $6 \%$ au cours de trois années et $5 \%$ au cours de quatre ou cinq des années considérées ( $c f$. infra).

Lorsque l'on observe la fréquence d'accès à la formation, uniquement parmi les seuls individus qui se sont formés, on constate un «effet de renforcement » pour certains groupes. Ainsi, globalement, les participants aux formations sont une majorité (58 \%) à y participer au cours de plus d'une année sur les cinq années observées. Ce résultat est fortement contrasté si l'on prend en compte le diplôme ou le statut professionnel. Comme l'illustre le tableau 2, les groupes qui accèdent en plus grand nombre à la formation semblent y participer, de surcroit, plus souvent. La plupart des cadres qui se forment $(40 \%)$, participent à au moins une forma- tion par $a^{10}$ durant trois années de la période d'observation ou davantage, tout comme $35 \%$ des diplômés de l'enseignement supérieur.

L'enquête de panel nous permet de tenir compte à la fois de la récurrence de la participation et de certaines caractéristiques des formations suivies. Nous avons ainsi pu distinguer différentes «pratiques de formation » (définies par la fréquence et le type de formation) au cours de la période étudiée (encadré 3).

Onze pratiques de formations ont ainsi pu être dégagées. Elles sont présentées dans le tableau 3. La pratique de formation la plus fréquente est la formation unique de courte durée «avec implication de l'employeur ». En revanche, lorsque les individus suivent plusieurs formations au cours de la période étudiée, les formations suivies, financées ou organisées par l'employeur, sont de durées variables.

Les individus qui participent à une formation, d'initiative personnelle, suivent soit une seule formation, soit à la fois des formations d'initiative personnelle et des formations à l'initiative de l'employeur («plusieurs formations diverses, sauf programmes d'emploi »).

Les formations développées dans le cadre des politiques de l'emploi sont suivies soit une fois soit sont associées au suivi d'autres types de formation.

${ }^{10}$ Comme nous le précisons dans l'encadré 2 , le questionnaire du panel ne permet pas de connaître le nombre de formations suivies sur une année. Tout au plus pouvons-nous savoir si l'individu a suivi au moins une formation au cours de l'année civile écoulée.

Tableau 2

Fréquence d'accès à la formation parmi l'ensemble des participants aux formations (\%)

\begin{tabular}{|l|c|c|c|c|}
\hline & $\begin{array}{c}\text { Formation au cours } \\
\text { d'une année }\end{array}$ & $\begin{array}{c}\text { Formation au cours de } \\
2 \text { années }\end{array}$ & $\begin{array}{c}\text { Formation au cours de } \\
3 \text { années ou + }\end{array}$ & Total \\
\hline Diplôme secondaire inférieur & 50 & 31 & 28 & 100 \\
\hline Diplôme enseignement supérieur & 37 & 28 & 35 & 100 \\
\hline Employés et ouvriers & 45 & 28 & 40 & 100 \\
\hline $\begin{array}{l}\text { Employés très qualifiés } \\
\text { ou cadres }\end{array}$ & 32 & 27 & 100 \\
\hline
\end{tabular}

(Chi2 significatif à .005 pour chaque croisement) $(*)$

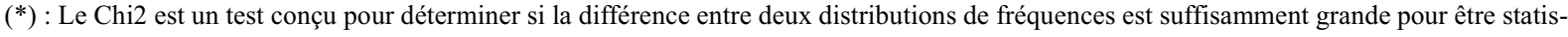
tiquement significative. Il permet d'établir la probabilité d'erreur de rejet de l'hypothèse nulle (aucune relation entre les deux variables).

Source : PSBH 1998-2002. 
Tableau 3

Pratiques de formation, parmi l'ensemble des participants aux formations au cours de la période 1997-2001 ( $\mathrm{N}=1.313)$ (*)

\begin{tabular}{|c|c|c|c|}
\hline & Pratiques de formation & $\begin{array}{c}\text { Nombre } \\
\text { d'individus }\end{array}$ & $\%$ \\
\hline $\begin{array}{l}\text { Une seule } \\
\text { formation }\end{array}$ & $\begin{array}{l}\text { Formation courte, avec implication de l'employeur } \\
\text { Formation longue, avec implication de l'employeur } \\
\text { Formation d'initiative personnelle } \\
\text { Formation dans le cadre d'un programme d'emploi } \\
\text { Non précisé }\end{array}$ & $\begin{array}{l}225 \\
131 \\
95 \\
70 \\
120\end{array}$ & $\begin{array}{l}17 \\
10 \\
7 \\
5 \\
9\end{array}$ \\
\hline $\begin{array}{c}\text { Plusieurs formations } \\
\text { de même type }\end{array}$ & $\begin{array}{l}\text { Formations courtes avec implication de l'employeur } \\
\text { Formations longues avec implication de l'employeur } \\
\text { Formations d'initiative personnelle } \\
\text { Formations dans le cadre de programmes d'emploi }\end{array}$ & $\begin{array}{l}130 \\
62 \\
45 \\
34\end{array}$ & $\begin{array}{c}10 \\
5 \\
3 \\
3\end{array}$ \\
\hline $\begin{array}{l}\text { Plusieurs formations } \\
\text { de types différents }\end{array}$ & $\begin{array}{l}\text { Formations avec implication de l'employeur, de durées variables } \\
\text { Plusieurs formations diverses sauf programme d'emploi } \\
\text { Plusieurs formations, surtout programmes d'emploi } \\
\text { Non précisé }\end{array}$ & $\begin{array}{l}182 \\
102 \\
78 \\
39\end{array}$ & $\begin{array}{l}14 \\
8 \\
6 \\
3\end{array}$ \\
\hline Total & & 1313 & 100 \\
\hline
\end{tabular}

$\left(^{*}\right)$ : La répartition selon les pratiques de formation est proposée - parmi les personnes qui ont complété le calendrier pour les cinq années d'observation 3290 individus ont été retenus - en tenant compte de celles qui ont suivi au moins une formation sur la période de cinq années considérées (1 313 au total).

Lecture : Parmi les 1313 individus qui ont suivi au moins une formation au cours des 5 années considérées, 225, soit $17 \%$, ont suivi une seule formation de courte durée et avec implication de l'employeur.

Source : PSBH ; calculs auteurs.

Finalement, les pratiques de formation les plus rares sont d'une part le suivi de plusieurs formations longues à l'initiative de l'employeur et, d'autre part, le suivi de plusieurs formations d'initiative personnelle ou dans le cadre de programmes d'emploi.

\section{L'INSCRIPTION DE LA FORMATION DANS DES TRAJECTOIRES PROFESSIONNELLES}

Étudier la participation à la formation continue en distinguant les caractéristiques individuelles des participants (sexe, âge, diplôme, etc.) s'avère intéressant dans la mesure où une partie des enjeux de la formation continue y sont liés. La prise en compte du niveau de formation initial peut, par exemple, s'inscrire dans un questionnement sur la «seconde chance » que pourrait constituer la participation à la formation continue pour les personnes qui sont entrées dans la vie active avec une faible qualification scolaire.

Toutefois, apprécier la participation à la formation au regard des seules caractéristiques individuelles comporte le risque de sur-valoriser l'initiative individuelle. Or, le contexte professionnel influence certainement autant la capacité et les opportunités réelles de suivre une formation que les caractéristiques personnelles.

En effet, dans un contexte où les trajectoires professionnelles sont fréquemment affectées par des mobilités contraintes et des épisodes de chômage, il nous a paru intéressant de chercher à préciser les liens entre les positions sur le marché du travail et les pratiques de formation continue. Notre hypothèse de 


\section{Encadré 3}

\section{Les « pratiques de formation continue »}

Afin de distinguer les différentes pratiques de formation, une construction a priori a été élaborée en distinguant successivement le nombre de formations suivies (une seule formation ou plusieurs formations), le type de formation lavec implication de l'employeur, d'initiative personnelle, dans le cadre d'un programme d'emploil et enfin, s'agissant des formations "avec implication de l'employeur », la durée (courtes si moins de 30 heures, longues si plus).

Cette catégorisation permet de préciser le type de formation suivie lorsqu'il s'agit d'un événement unique, ou, lorsque plusieurs formations sont suivies, de distinguer les cas de participations successives à un même type de formation (par exemple, plusieurs formations suivies d'initiative personnelle ou plusieurs formations avec implication de l'employeur) et ceux où plusieurs formations de types différents se succèdent (par exemple, plusieurs formations avec implication de l'employeur mais de durées variables).

départ était que, selon les trajectoires professionnelles, les opportunités de suivre une formation sont variables. Ainsi, à âge ou niveau de qualification égal, deux individus n'auront pas les mêmes opportunités selon qu'ils sont, par exemple, stabilisés en emploi depuis plusieurs années ou qu'ils alternent emplois et épisodes de chômage dans des trajectoires instables. Ces éléments de contexte peuvent aussi déterminer le type de formation suivie (implication de l'employeur ou pas, programme de réinsertion de demandeurs d'emploi, etc.). Les pratiques de formation s'inscrivent ainsi dans un espace d'opportunités largement dépendant de la situation des individus sur le marché du travail. Nous présentons d'abord les différents types de trajectoires professionnelles que nous avons pu distinguer, puis les caractéristiques de la participation à la formation des individus qui s'y sont inscrits.

\section{Trajectoires professionnelles}

Différentes trajectoires professionnelles ont été construites à partir des statuts occupés aux différents moments de l'enquête et des transitions possibles entre ces statuts (encadré 4).

Six ensembles cohérents de trajectoires ont pu être dégagés par l'analyse des données (encadré 4). Les trajectoires se définissent par des similitudes en termes de transitions ou de permanence de statuts. Les différentes trajectoires observées sont présentées dans le tableau $4^{11}$. Comme nous le verrons, les trajectoires professionnelles reflètent pour une part la stabilité (ou l'instabilité) dans l'emploi qui est un élément déterminant de la participation à la formation continue.

La trajectoire quantitativement la plus représentée se caractérise par la prédominance de l'emploi sur la période observée $(80 \%$ des individus de ce groupe sont en emploi sans discontinuité et $97 \%$ sont à l'emploi durant au moins quatre des cinq années). Une autre trajectoire caractérisée par la permanence des statuts est celle de l'enlisement dans le chômage. Les individus relevant de cette trajectoire ont passé la plus grande partie de la période au chômage (tous au moins trois ans et $87 \%$ durant quatre ans ou plus; de brefs épisodes d'emploi peuvent donc s'inscrire dans ces parcours). Un troisième ensemble de trajectoires se caractérise par l'inactivité sur l'ensemble de la période. Trois types de situations peuvent être ici distingués. L'un est dominé par le statut de femme au foyer («ménagère »); le deuxième parcours est celui des (pré)pensionnés; le dernier concerne les jeunes aux études durant toute la période.

Les trois derniers groupes de parcours sont davantage marqués par des entrées et sorties du marché du travail ou des transitions entre emploi et chômage et/ ou inactivité. Ainsi, le groupe des trajectoires précaires se définit par des transitions diverses entre emploi et chômage et donc par l'instabilité de l'insertion professionnelle. Les individus composant ce groupe passent, pour la plupart, environ la moitié de la période en emploi et l'autre moitié au chômage. La plupart du temps, ce parcours se compose d'une succession de différents brefs épisodes d'emploi et

\footnotetext{
11 Voir Cardelli et al. (2005) pour davantage de détails.
} 


\section{Encadré 4 \\ La construction de trajectoires professionnelles}

Le PSBH (Panel Survey on Belgian Household) renseigne sur les statuts occupés (en emploi, étudiant, chômeur, (pré-) pensionné, au foyer) lors de chaque mois de l'année considérée à chacune des vagues du panel. II nous a ainsi été possible de reconstituer le calendrier des parcours pour la période du 1er janvier 1997 au 31 décembre 2001. Au total, des parcours ont pu être distingués pour 3290 individus ( ${ }^{*}$ ) de notre échantillon. Ce sous-échantillon correspond aux hommes âgés de moins de 65 ans et aux femmes âgées de moins de 60 ans au 31 décembre $20011^{* *}$ ). II s'agit par ailleurs des personnes ayant complété leur calendrier à chacune des cinq vagues.

Le tableau des soixante variables indiquant le statut occupé mois par mois est soumis à une analyse factorielle des correspondances (variables « actives » dans l'analyse), puis à une classification ascendante hiérarchique. Cette procédure permet de regrouper les individus qui se trouvent le plus souvent dans les mêmes statuts aux mêmes moments. Les regroupements sont effectués sur la base des statuts occupés aux différentes dates. Dans un second temps, un ensemble de variables individuelles (sexe, âge, diplôme) est utilisé pour l'interprétation de ces trajectoires personnelles. Ces dernières variables sont considérées comme "illustratives» dans l'analyse factorielle. Elles ne participent pas à la construction des trajectoires, mais elles peuvent être utilisées pour décrire les groupes constitués. Les variables relatives à la participation à la formation peuvent également être analysées comme variables illustratives.

L'analyse des données nous a conduits à distinguer onze trajectoires types. Dans un second temps, nous avons choisi d'en regrouper certaines relativement similaires, pour ne plus retenir que six ensembles de trajectoires. Nous avons par exemple regroupé plusieurs trajectoires « hors travail » (étude, inactivité des femmes) en une seule trajectoire " d'inactivité ».

$(*)$ : En effet, parmi les 3509 individus de l'échantillon initial, seuls ceux qui ont complété le calendrier pour les cinq années d'observation ont été retenus, soit 3290 personnes.

(**) : L'âge légal de retraite des femmes en 2001 en Belgique était de 60 ans.

Tableau 4

Types de parcours professionnels observés entre 1997 et 2001 ( $N=3.290$ )

\begin{tabular}{|l|l|c|c|}
\hline \multicolumn{1}{|c|}{ Parcours } & \multicolumn{1}{c|}{ Caractéristiques } & $\begin{array}{c}\text { Nombre } \\
\text { d'individus }\end{array}$ & \% \\
\hline Stabilité dans l'emploi & Trajectoire largement dominée par l'emploi & 2258 & 69 \\
\hline Inactivité & $\begin{array}{l}\text { Femmes au foyer (" ménagères »), (pré)pensionnés, } \\
\text { étudiants }\end{array}$ & 497 \\
\hline Trajectoires précaires & $\begin{array}{l}\text { Alternance de situations d'emploi, de chômage, } \\
\text { d'inactivité }\end{array}$ & 159 & 5 \\
\hline Entrées sur le marché du travail & Transitions études - emploi & 142 & 139 \\
\hline Chômage & Chômage dominant la période & 4 \\
\hline Sorties du marché du travail & $\begin{array}{l}\text { Passage à la (pré)pension ou en inactivité en fin de } \\
\text { période }\end{array}$ & $\mathbf{9 5}$ \\
\hline Total & & $\mathbf{3} \mathbf{2 9 0}$ & $\mathbf{1 0 0}$ \\
\hline
\end{tabular}

Source : PSBH ; calculs auteurs. 
de chômage. Notre échantillon comprend une partie de jeunes entrant sur le marché du travail. Leurs trajectoires se caractérisent par le passage des études vers l'emploi moyennant, pour certains, un ou plusieurs épisodes courts de chômage. Enfin, le dernier groupe de parcours est défini par les sorties $d u$ marché $d u$ travail. On peut y distinguer deux sous-groupes. Le premier concerne les partants vers la (pré)pension et le second comprend des trajectoires déterminées par une période d'inactivité.

Schématiquement, on distingue donc deux grands types de parcours : les premiers se caractérisent par une distance forte ou un retrait du marché du travail (trajectoires de chômage, d'inactivité ou de sortie en fin de carrière) et les seconds se définissent par une plus grande participation à l'emploi, soit en début de vie active (entrants) ou en cours de carrière, avec une intensité d'emploi plus ou moins fréquente (trajectoires stables ou précaires). Nous présentons cidessous les données relatives à la participation à la formation de ces différents groupes.

\section{Trajectoires professionnelles et différenciation des pratiques de formation}

Un premier constat porte sur l'accès restreint à la formation dans les trajectoires que l'on pourrait qualifier de «moindre participation à l'emploi ». En effet, moins une trajectoire comporte d'épisodes d'emploi, moins la participation à la formation sera élevée. Ce constat peut être établi pour trois groupes de trajectoires : les trajectoires d'inactivité (moins de $10 \%$ des individus ont participé à la formation au cours de la période étudiée; sur base annuelle, le taux n'atteint jamais 3\%), les trajectoires de chômage (groupe composé pour deux tiers de femmes et majoritairement de peu qualifiés, où la participation n'atteint pas $18 \%$ sur la période et est rarement récurrente), et, enfin, les trajectoires de sortie du marché du travail où le niveau de participation à la formation, déjà faible en début de période (alors que les individus sont encore à l'emploi), rejoint vers la fin de la période celui des inactifs.

A contrario, une partie des trajectoires caractérisées par la participation au marché du travail est associée à une participation plus forte aux formations profes- sionnelles. Mais plus que par l'accès à la formation, c'est par la récurrence de cette démarche que les différences se marquent.

Ainsi, les jeunes entrant sur le marché du travail participent davantage à la formation $(53 \%$ sur la période), mais aussi relativement plus souvent (la moitié des formés se forment au moins au cours de deux années sur cinq). Il en va presque de même pour les parcours caractérisés par la stabilité dans l'emploi : la participation à la formation y est relativement élevée (49\% des individus participent à la formation sur la période étudiée) et plutôt récurrente. Néanmoins, nous montrerons que ce groupe des travailleurs stables, quantitativement important, présente une forte hétérogénéité qui peut être associée à des pratiques de formation différenciées (cf. infra).

Le niveau de participation des travailleurs dont les trajectoires ont été qualifiées de précaires se situe entre celui des chômeurs et celui des personnes stabilisées en emploi $(35 \%$ au cours de la période observée). Parmi les formés de ce groupe, les retours en formation sont fréquents.

En résumé, plus le lien au marché du travail semble fort, plus l'accès à la formation est élevé et plus la participation à la formation est récurrente. Il y a toutefois lieu d'interpréter avec prudence cette relation. Il convient en effet de prendre en compte d'autres variables (genre, diplôme, etc.) qui peuvent être associées à la trajectoire. Une analyse plus détaillée des trajectoires professionnelles selon le niveau de diplôme, le sexe et l'âge met en évidence quelques traits de sélectivité du marché du travail. En particulier, parmi les jeunes, la difficulté à se stabiliser en emploi concerne essentiellement les plus bas niveaux de diplôme. Par ailleurs, les moments et les formes de retrait du marché du travail se différencient très nettement selon le sexe, et pour les femmes, selon le niveau de diplôme obtenu. Ainsi, au travers des possibilités d'accès ou de maintien dans l'emploi, le niveau de diplôme continue à jouer un rôle important et conditionne la participation à la formation professionnelle.

En outre, on observe que l'inégalité d'accès ou d'intensité de la participation à la formation se 
Tableau 5

Accès à la formation selon le type de parcours professionnel entre 1997 et 2001 (\%)

\begin{tabular}{|l|c|c|c|}
\hline \multicolumn{1}{|c|}{$\begin{array}{c}\text { Trajectoires } \\
(\mathbf{N}=3.290)\end{array}$} & $\begin{array}{c}\text { Accès période } \\
1997-2001\end{array}$ & $\begin{array}{c}\text { dont au cours } \\
1 \text { an s/5 }\end{array}$ & $\begin{array}{c}\text { dont au cours } \\
2 \text { ans s/5 }\end{array}$ \\
\hline Entrées sur le marché du travail & 53 & 27 & 17 \\
\hline Stabilité dans l'emploi & 49 & 19 & 14 \\
\hline Trajectoires précaires & 35 & 13 & 16 \\
\hline Sorties du marché du travail & 24 & 15 & 5 \\
\hline Chômage & 18 & 8 & 5 \\
\hline Inactivité & 9 & 8 & 1 \\
\hline Ensemble échantillon & 40 & 17 & 17 \\
\hline
\end{tabular}

Lecture : Parmi les individus entrant dans l'emploi, $53 \%$ ont suivi au moins une formation au cours de la période étudiée (1997-2001); ils étaient $27 \%$ à avoir été en formation au cours d'une seule des cinq années et $17 \%$ à avoir accédé à la formation au cours de deux années.

Source : PSBH ; calculs auteurs.

double d'une différenciation liée aux cadres institutionnels dans lesquels les formations sont développées. Si, dans l'ensemble, les formations organisées par l'employeur, de courtes durées, sont les plus représentées dans l'échantillon (cf. supra), elles le sont principalement au sein des trajectoires stables ou de sortie.
Ainsi, si l'on s'intéresse aux seuls participants aux formations, parmi les pratiques de formation identifiées, on distingue les formations organisées ou financées par l'employeur, au sein de l'entreprise, qui profitent surtout aux entrants, aux travailleurs stables et, dans une moindre mesure, aux sortants, et celles développées dans le cadre de politiques publi-

Tableau 6

Pratiques de formation des individus formés selon le type de parcours professionnel entre 1997 et 2001 (en \% des individus de la trajectoire)

\begin{tabular}{|c|c|c|}
\hline Trajectoires & Types de formation les plus fréquents & $\%$ trajectoire \\
\hline $\begin{array}{l}\text { Stabilité dans l'emploi } \\
(\mathrm{N}=2258)\end{array}$ & Plusieurs formations avec implication de l'employeur & 37 \\
\hline $\begin{array}{l}\text { Inactivité } \\
(\mathrm{N}=497)\end{array}$ & \begin{tabular}{|l} 
Formation d'initiative personnelle \\
Formation dans le cadre d'un programme d'emploi
\end{tabular} & $\begin{array}{l}47 \\
24\end{array}$ \\
\hline $\begin{array}{l}\text { Précaire } \\
(\mathrm{N}=159)\end{array}$ & $\begin{array}{l}\text { Plusieurs formations dans le cadre d'un programme d'emploi } \\
\text { Une formation programmes d'emploi et une formation } \\
\text { employeur }\end{array}$ & $\begin{array}{l}22 \\
18\end{array}$ \\
\hline $\begin{array}{l}\text { Entrée sur le marché du travail } \\
(N=142)\end{array}$ & $\begin{array}{l}\text { Plusieurs formations impliquant l'employeur } \\
\text { Une seule formation, longue, impliquant l'employeur } \\
\text { Une formation d'initiative personnelle }\end{array}$ & $\begin{array}{l}24 \\
23 \\
15\end{array}$ \\
\hline $\begin{array}{l}\text { Chômage récurrent } \\
(N=139)\end{array}$ & $\begin{array}{l}\text { Une formation dans le cadre de programmes d'emploi } \\
\text { Plusieurs formations dans le cadre de programmes d'emploi }\end{array}$ & $\begin{array}{l}25 \\
25\end{array}$ \\
\hline $\begin{array}{l}\text { Sortie marché du travail } \\
(N=95)\end{array}$ & Une formation (courte) avec implication employeur & 26 \\
\hline
\end{tabular}

Lecture : Au sein des trajectoires précaires, les types de formation les plus fréquents sont les formations d'initiative personnelle (47\% des formations suivies par les individus inscrits dans cette trajectoire) et les formations développées dans le cadre d'un programme d'emploi (24 \%).

Source : PSBH ; calculs auteurs. 
ques de formation qui s'adressent surtout aux inactifs, aux travailleurs précaires et aux chômeurs. Pour les précaires, l'accès à l'emploi implique rarement un accès à la formation dans le cadre de l'emploi. Enfin, les formations suivies d'initiative personnelle le sont en plus grande proportion par les inactifs, les chômeurs ou les jeunes entrants sur le marché du travail. Le tableau 6 présente, pour chaque trajectoire identifiée, les pratiques de formation les plus fréquentes.

\section{LES TRAVAILLEURS STABLES : UN GROUPE HÉTÉROGĖNE, DES PRATIQUES DE FORMATION DIVERSIFIÉES}

Les trajectoires de stabilité dans l'emploi concernent des personnes qui passent l'essentiel de la période de cinq années en emploi (éventuellement chez plusieurs employeurs). Ces trajectoires sont de loin les plus fréquentes et se caractérisent par un usage assez intense de la formation professionnelle. Cependant, les parcours professionnels stables recouvrent des situations d'emploi très diverses. Ces trajectoires stables peuvent être associées à une forte intégration professionnelle ou au contraire à des formes de précarité qui touchent les dimensions de l'emploi et/ ou du travail (elles recouvrent aussi bien des parcours de cadres hautement qualifiés que des travailleurs appartenant au «noyau dur» du salariat, ou encore de salarié(e)s à temps partiel et bas salaire). Il nous a dès lors paru intéressant de préciser les pratiques de formation des travailleurs stabilisés en emploi, en distinguant ceux-ci sur la base de plusieurs caractéristiques des emplois qu'ils ont occupés (encadré 5).

\section{Une partition statistique des «travailleurs stables»?}

À partir des dimensions retenues pour différencier les positions occupées par les travailleurs stables, quatre situations d'emploi ont pu être identifiées; elles sont présentées dans le tableau 7.

\section{Encadré 5}

Situations d'emploi au sein des trajectoires stables

Pour étudier les situations d'emploi des travailleurs stabilisés sur le marché du travail, nous avons sélectionné les individus salariés aux cinq moments d'interrogation du panel (printemps de chaque année), soit 1787 individus $\left.{ }^{*}\right)$.

Le regroupement des situations d'emploi a été réalisé au moyen d'une analyse de correspondance s'appuyant sur huit variables relatives aux emplois occupés en 1998, prolongée par une classification ascendante hiérarchique (CAH). Les huit variables sont : le régime horaire (temps plein, temps partiel), la réalisation d'heures supplémentaires (2 heures ou moins, 3 à $10 \mathrm{~h}$, plus de $10 \mathrm{~h}$ par semaine), le salaire (mensuel net réparti en huit classes converties en euros), la catégorie socioprofessionnelle louvrier non qualifié, ouvrier qualifié, employé, employé à haute qualification ou cadre, autre catégorie), la satisfaction à l'égard du revenu, du nombre d'heures de travail, du contenu du travail et des conditions de travail (pour ces quatre variables, quatre modalités sont distinguées: très insatisfait, insatisfait, satisfait, très satisfait).

Sur la base de ces variables et modalités actives dans l'analyse factorielle, nous avons retenu le découpage en huit classes (plus celle associée à des données incomplètes) issu de la $\mathrm{CAH}$ et nous avons procédé à des regroupements pour retenir finalement quatre groupes (car, comme pour l'analyse des trajectoires, certains groupes distincts du point de vue du codage des variables, présentaient des similitudes du point de vue de notre interprétation).

$\left(^{*}\right)$ : Ces individus composent un sous-échantillon plus restrictif dans l'ensemble des travailleurs aux trajectoires stables dans la mesure où ne sont plus pris en compte les indépendants et les individus qui seraient passés par une courte période de chômage dans la période de référence pour au moins une des cinq années considérées. 
Tableau 7

Groupes de salariés inscrits dans une trajectoire stable $(N=1.787)$

\begin{tabular}{|c|l|c|}
\hline Groupe & \multicolumn{1}{|c|}{ Type d'emploi occupé en 1998 } & \% des trajectoires stables \\
\hline A & "Temps partiels, bas salaires » & 11 \\
\hline B & "Salaires moyens, insatisfaits » & 16 \\
\hline C & "Temps pleins, salaires moyens, satisfaits » & 46 \\
\hline D & "Cadres » & 22 \\
\hline (e) & (données incomplètes) & (7) \\
\hline
\end{tabular}

Source : PSBH 1998-2002, calculs auteurs.

Le premier groupe (A) de travailleurs stables est caractérisé par les contrats de travail à temps partiel et un bas niveau de rémunération. Il s'agit essentiellement de femmes (93\%), relativement moins diplômées que la moyenne de l'échantillon, employées ou ouvrières non qualifiées. Le travail à temps partiel n'est pas compensé par les heures supplémentaires. Leurs salaires sont faibles. La satisfaction au travail est importante dans ce groupe, surtout par rapport aux horaires, aux conditions de travail et au nombre d'heures de travail hebdomadaires; elle l'est peu par rapport au salaire ${ }^{12}$.

Le deuxième groupe (B) se caractérise par une grande insatisfaction à l'égard du travail. Cette insatisfaction porte sur les conditions et le nombre d'heures de travail mais concerne surtout le salaire. On trouve ici davantage d'hommes (56\%) que de femmes, se répartissant dans deux types de statuts professionnels : d'une part, les employés, contraints de réaliser des heures supplémentaires et, d'autre part, des ouvriers qualifiés. Ce groupe est légèrement plus présent dans les régions wallonne et bruxelloise ; on y trouve aussi une part significative de diplômés de l'enseignement supérieur (près de $40 \%$ ). Sans doute, des pratiques de déclassement à l'embauche expliquent-elles cette forte insatisfaction au travail. En effet, la plupart de ces salariés (72\%) estime « disposer de compétences ou de qualifications suffisantes pour exercer une activité professionnelle plus exigeante $»$.

${ }^{12} \mathrm{Si}$ la satisfaction est clairement exprimée sur ces aspects, on peut toutefois supposer que la prise en compte des contraintes temporelles hors travail (et l'intériorisation de rôles féminins) détermine assez largement ces réponses. En outre, cette satisfaction générale est tempérée par un jugement plus timoré à l'égard du salaire.
D'autres salariés, les plus nombreux, manifestent une forte satisfaction à l'égard du travail. Le troisième groupe (C) est constitué de ce que l'on surnomme parfois le «noyau dur» du salariat. Il s'agit de salariés travaillant à temps plein, ne réalisant pas d'heures supplémentaires, satisfaits du contenu, des horaires de travail et de leur rémunération. Les ouvriers qualifiés sont surreprésentés dans ce groupe.

Enfin, le dernier groupe (D) occupe les positions privilégiées du marché du travail. Il s'agit essentiellement de cadres, à haut niveau de rémunération. Majoritairement masculins (71\%) et diplômés de l'enseignement supérieur ( $80 \%$ ), ils sont aussi plus nombreux à appartenir à la classe d'âges [45-54 ans]. Ces individus se déclarent (très) satisfaits de leur travail, surtout en ce qui concerne le type de travail réalisé et le revenu. Ils se disent satisfaits (plus rarement très satisfaits) des horaires, malgré un nombre important d'heures supplémentaires.

\section{Quelles pratiques de formation dans les trajectoires stables?}

Nous avons souligné combien une trajectoire stable constituait un contexte favorable à la participation à la formation continue, sans toutefois en constituer une garantie. Ainsi, même les groupes (A et B) occupant les emplois les moins attractifs parmi les stables participent davantage à la formation que les individus inscrits dans des trajectoires précaires ou de chômage.

On constate toutefois de fortes différences de participation entre groupes de travailleurs stables (de $40 \%$ pour les bas salaires et temps partiels à près de $65 \%$ pour les cadres). Si l'on s'intéresse à la récurrence de 
Tableau 8

Participation à la formation des différents groupes de travailleurs stables

\begin{tabular}{|c|l|c|c|c|}
\hline Groupe & \multicolumn{1}{|c|}{ Type d'emploi occupé en 1998 } & $\begin{array}{c}\text { Formation entre } \\
1997 \text { et 2001 }\end{array}$ & $\begin{array}{c}\text { Formation au } \\
\text { cours de } \\
2 \text { années }\end{array}$ & $\begin{array}{c}\text { Formation au } \\
\text { cours de } \\
3 \text { années }\end{array}$ \\
\hline A & "Temps partiels, bas salaires & $41 \%$ & $11 \%$ & $9 \%$ \\
\hline B & "Salaires moyens, insatisfaits & $47 \%$ & $12 \%$ & $13 \%$ \\
\hline C & "Temps pleins, salaires moyens, satisfaits» & $49 \%$ & $14 \%$ & $16 \%$ \\
\hline D & "Cadres» & $65 \%$ & $19 \%$ & $23 \%$ \\
\hline
\end{tabular}

Source : PSBH 1998-2002 Chi2 sig à 0.05 pour chaque croisement.

la formation, on observe que les retours en formation ne s'opèrent que pour un cinquième à un quart de la plupart des travailleurs stables, à l'exception des cadres qui sont plus de $40 \%$ à se former fréquemment. De ce point de vue, même si les cinq années considérées ne rendent compte que d'une période limitée de la vie active, la « formation tout au long de la vie » n'apparaît être une réalité que pour ce dernier groupe de travailleurs.

Si l'on mesure l'accès et la récurrence de la formation pour les différents groupes de travailleurs stables, on observe que la participation à la formation tend à s'accroître lorsque la position sur le marché du travail s'améliore. Ainsi, plus le salaire et la satisfaction au travail augmentent, plus la participation et la récurrence seront élevées. Le groupe des cadres se distingue en outre par une participation nettement plus élevée et plus fréquente (tableau 8) que celle observée dans les autres groupes : si l'on compare les deux groupes extrêmes, on constate que les cadres accèdent 1,6 fois plus à la formation que les travailleurs stables à temps partiel et bas salaires ou que les cadres participant à une formation au cours de trois années sont 2,7 fois plus nombreux que ces derniers.

Une analyse plus fine ne permet pas de distinguer les différents sous-groupes de travailleurs stables à partir des variables qui nous ont permis d'identifier les différentes pratiques de formation (durée de la formation, lien avec un programme d'emploi, initiative personnelle, implication de l'employeur, etc.). Les groupes que nous avons distingués au sein des trajectoires stables se différencient donc davantage des autres types de trajectoire par la récurrence dans la participation à la formation professionnelle que par la variété des pratiques de formation ${ }^{13}$.

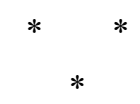

Les pratiques de formation des travailleurs identifiées à partir des données du PSBH (Panel Survey on Belgian Household) permettent de souligner certains traits d'une segmentation importante des pratiques de formation professionnelle continue.

Nos analyses suggèrent une distinction de l'offre de formation entre d'une part, celle reposant sur une implication des entreprises et qui s'adresse en priorité aux travailleurs les mieux insérés et intégrés professionnellement et, d'autre part, celle reposant plus exclusivement sur l'action des pouvoirs publics (programmes d'emploi, voire une partie des formations sans implication de l'employeur) qui s'adresse davantage aux travailleurs moins ou non insérés sur le marché du travail. Les personnes durablement

\footnotetext{
${ }^{13}$ Les travailleurs stables participent essentiellement à des formations organisées par l'employeur. On constate toutefois que les individus appartenant aux deux premiers groupes (bas salaires ou insatisfaits) participent un peu plus fréquemment que les autres travailleurs « stables » aux formations sur initiative personnelle ou dans le cadre des programmes d'emploi. Nos données, portant sur un nombre réduit d'observations, montrent ainsi que les formations sur initiative personnelle représentent $7 \%$ des formations de l'ensemble des travailleurs stables pour $11 \%$ chez les travailleurs insatisfaits et $15 \%$ chez les travailleurs (travailleuses) à temps partiel. Les formations développées dans le cadre d'un programme d'emploi représentent $9 \%$ des pratiques de formation chez les travailleurs stables et $14 \%$ chez les travailleurs qui expriment le plus d'insatisfaction au travail. Les cadres, pour leur part, pratiquent davantage de retours en formations organisées ou soutenues par l'employeur (50\% des formations des cadres pour $39 \%$ des formations pour l'ensemble des travailleurs stables).
} 
touchées par le chômage, mais aussi les travailleurs qui occupent des emplois de manière discontinue accèdent moins aux formations, et lorsqu'ils y accèdent, ces formations ne sont ni financées ni organisées par les employeurs.

Alors que les dernières décennies ont été marquées par un accroissement de la précarité des trajectoires, les usages de la formation par les entreprises, en particulier au travers de la sélection des travailleurs appelés à y participer, semblent corroborer, voire renforcer la segmentation du marché du travail. Cet aspect qui ressort de l'enquête généraliste PSBH mériterait d'être plus spécifiquement étudié à partir de données mettant davantage en lumière les parcours marqués par l'instabilité de l'emploi.

Les travailleurs les mieux insérés professionnellement accèdent plus souvent aux formations, et ce dans le cadre de l'entreprise. Même s'il s'agit souvent de formations courtes, elles participent à une socialisation à l'univers de l'entreprise, dont ne bénéficient pas les travailleurs moins bien insérés. Ce déficit peut-il être compensé par l'accès à d'autres formations, notamment celles organisées dans le cadre de programmes d'emploi ? Les politiques d'insertion et de formation, qui s'inscrivent aujourd'hui dans des objectifs de développement de " l'employabilité » et de la «flexicurité » devraient intégrer ces réflexions.

À partir des données du PSBH, nous nous sommes également interrogés sur les formes de segmentation dans la participation aux formations des travailleurs stabilisés en emploi. Des clivages ressortent de l'analyse, opposant les salariés les mieux intégrés professionnellement (dont le «cadre » apparaît comme figure emblématique) aux autres travailleurs. Par ailleurs, parmi les travailleurs stables, l'accès à la formation et l'intensité de cet accès discrimineraient davantage les différents groupes de travailleurs que les caractéristiques organisationnelles des formations, notamment la durée des formations.

Cette approche exploratoire du panel nous a permis d'établir quelques constats relatifs aux pratiques de formation et à leur inscription dans les parcours professionnels. Elle n'épuise certainement pas les ressources qu'une enquête de panel, même généraliste comme le PSBH, offre pour l'étude de la participation à la formation. En effet, il serait intéressant de comprendre dans quelle mesure celle-ci peut être structurée par d'autres dimensions que celles qui ont été prises en compte ici, par exemple, le revenu des ménages, la situation familiale ou d'autres aspects des parcours professionnels. Au-delà d'une exploitation plus complète de ce type de données, il nous semble aussi nécessaire de recourir à d'autres méthodologies pour mieux comprendre comment les pratiques de formation s'articulent aux usages des formations par les entreprises, dans le cadre de leur gestion du personnel. Le recours aux données du panel souligne en tous cas la pertinence d'une approche longitudinale de la formation, en particulier pour explorer davantage les formes prises par la participation aux formations professionnelles; et ce, dans un contexte qui valorise fortement l'implication des travailleurs, notamment au travers de leur participation à la formation, mais où cette dernière demeure une ressource inégalement répartie entre les composantes de la population active.

Bibliographie

Baye A., Demeuse M., Hendrickx G., Mainguet Ch. (2003), « La formation continue en Belgique: profil des participants et esquisses de parcours. Exploitation de l'Enquête sur les forces de travail
2000 », in Guyot J.-L., Mainguet C., Van Haeperen B., La formation professionnelle continue. L'individu au cour des dispositifs, Éditions De Boeck, Bruxelles. 
Cardelli R., Conter B., Veinstein M. (2005), "L'inscription de la formation continue dans les trajectoires professionnelles. Une analyse exploratoire de l'enquête sur le panel belge des ménages (1997-2001) », in Giret J.-F, Grelet Y., Ourtau M., Werquin P. (Eds), Construction et validation des compétences: l'apport des analyses longitudinales (actes des XII journées du Longitudinal), Relief $\mathrm{n}^{\circ} 8$, Céreq, Marseille.

Conter B., Maroy C., Urger F. (1999), Le développement de la formation professionnelle continue en Belgique francophone, Cahier de la recherche $d u$ GIRSEF, $\mathrm{n}^{\circ}$ 3, UCL, Louvain-la-Neuve.

De Brier C., Legrain A. (2002), Politiques de formation dans les entreprises. La situation belge en chiffres 1999, Ichec, Bruxelles.

Doutrelepont R., Mortelmans R., Casman M.-T. (2004), Onze ans de vie en Belgique. Analyse socioéconomiques à partir du Panel de Démographie Familiale, Gent, Academia Press.
Dubar Cl. (2004), La formation professionnelle continue, $5^{\mathrm{e}}$ édition, La Découverte, Paris.

Heuze P., Zachary M.-D. (2003), «La formation continue dans les entreprises dans les bilans sociaux », Lettre d'information du TEF, ${ }^{\circ} 1-3$, ULB, Bruxelles.

Monville M. (2007), Évaluation des efforts de formation professionnelle continue, Note du Conseil central de l'économie 2007-855, mimeo.

Monville M., Léonard D. (2008), «La formation professionnelle continue », Courrier hebdomadaire $\mathrm{n}^{\circ}$ 1987-1988, CRISP, Bruxelles.

Nester K., Kailis E. (2002), « La formation continue en entreprise dans l'Union européenne et en Norvège (CVTS 2)», Statistiques en Bref, Eurostat, $\mathrm{n}^{\circ} 3 /$ 2002.

Trautmann J. (2003), «Accès et retours à la formation », Formation Emploi $\mathrm{n}^{\circ} 1$.

\section{Résumé}

\section{Formation professionnelle continue en Belgique : Des pratiques variables selon les trajectoires professionnelles}

L'article présente une exploitation du Panel belge de démographie familiale pour étudier le lien entre trajectoires professionnelles et participation à la formation. Après avoir exposé rapidement quelques données sur la participation des adultes à la formation en Belgique, il propose une première approche descriptive portant sur la fréquence d'accès à la formation et la récurrence de cette participation. Dans un deuxième temps, une typologie de trajectoires professionnelles est élaborée et croisée avec différentes pratiques de formation. Enfin, la participation à la formation des travailleurs "stabilisés dans l'emploi » est plus particulièrement analysée.

Mots clés

Belgique, formation professionnelle, cheminement professionnel Journal of Economic Literature: M 53, J 24 